\title{
Bonding Heterogeneity in Mixed-Anion Compounds Realizes Ultralow Lattice Thermal Conductivity
}

Naoki Sato, * Norihide Kuroda, Shun Nakamura, Yukari Katsura, Ikuzo Kanazawa, Kaoru Kimura, and Takao Mori

Dr. N. Sato

International Center for Young Scientists (ICYS), National Institute for Materials Science (NIMS)

1-2-1 Sengen, Tsukuba, Ibaraki 305-0047, Japan

E-mail: SATO.Naoki@nims.go.jp.

Dr. N. Sato, Prof. Dr. T. Mori

International Center for Materials Nanoarchitectonics (WPI-MANA), NIMS

1-1 Namiki, Tsukuba, Ibaraki 305-0047, Japan

Mr. N. Kuroda, Mr. S. Nakamura, Prof. Dr. I. Kanazawa

Department of Physics, Tokyo Gakugei University

4-1-1 Nukuikitamachi, Koganei, Tokyo 184-8501, Japan

Dr. Y. Katsura

Research and Services Division of Materials Data and Integrated System (MaDIS), NIMS

1-1 Namiki, Tsukuba, Ibaraki 305-0047, Japan

Prof. Dr. K. Kimura, Dr. Y. Katsura

Department of Advanced Materials Science, The University of Tokyo

5-1-5 Kashiwanoha, Kashiwa, Chiba 277-8561, Japan

Prof. Dr. K. Kimura

OPERANDO-OIL, National Institute of Advanced Industrial Science and Technology (AIST) 5-1-5 Kashiwanoha, Kashiwa, Chiba 277-8561, Japan

Prof. Dr. T. Mori

Graduate School of Pure and Applied Science, University of Tsukuba

1-1-1 Tennodai, Tsukuba, Ibaraki 305-8671, Japan

Keywords: ab initio calculation, bonding heterogeneity, lattice dynamics, lattice thermal conductivity, mixed-anion compound

Crystalline materials with intrinsically low lattice thermal conductivity ( $\kappa_{\text {lat }}$ ) pave the way towards high performance in various energy applications, including thermoelectrics. Here we demonstrate a strategy to realize ultralow $\kappa_{\text {lat }}$ using mixed-anion compounds. Our calculations reveal that locally distorted structures in chalcohalides $\mathrm{MnPnS}_{2} \mathrm{Cl}(\mathrm{Pn}=\mathrm{Sb}, \mathrm{Bi})$ derives a bonding heterogeneity, which in turn causes a peak splitting of the phonon density of states. This splitting induces a large amount of scattering phase space. Consequently, $\kappa_{\text {lat }}$ of $\mathrm{MnPnS}_{2} \mathrm{Cl}$ 
is significantly lower than that of a single-anion sulfide $\mathrm{CuTaS}_{3}$ with a similar crystal structure. Experimental $\kappa_{\text {lat }}$ of $\mathrm{MnPnS}_{2} \mathrm{Cl}$ takes an ultralow value of about $0.5 \mathrm{~W} \mathrm{~m}^{-1} \mathrm{~K}^{-1}$ at $300 \mathrm{~K}$. Our findings will encourage the exploration of thermal transport in mixed-anion compounds, which remain a vast unexplored space, especially regarding unexpectedly low $\kappa_{\text {lat }}$ in lightweight materials derived from the bonding heterogeneity.

\section{Introduction}

Searches for low lattice thermal conductivity $\left(\kappa_{\text {lat }}\right)$ materials have been of great importance in achieving high performance in thermoelectrics, ${ }^{[1-3]}$ thermal barrier coatings,${ }^{[4]}$ and thermal insulation. ${ }^{[5]}$ In the past decade, the validity of some extrinsic effects for reducing $\kappa_{\text {lat }}$, such as point defects, ${ }^{[6,7]}$ fine grain boundary, ${ }^{[8]}$ nanoscale precipitates, ${ }^{[9]}$ and all-scale hierarchical microstructures, ${ }^{[10]}$ has been successfully demonstrated. However, these strategies usually affect charge carrier mobility, which is detrimental to thermoelectric performance. Thus, understanding the intrinsic mechanisms of thermal transport realizing low $\kappa_{\text {lat }}$ which are associated closely with chemical bonding, crystal structure, and lattice anharmonicity, can be more helpful in some cases, as it provides us with efficient ways to manage both electrical and thermal properties independently.

In recent years, several structural origins bringing intrinsically low $\kappa_{\text {lat }}$ like complex crystal structure ${ }^{[11-20]}$ resonant bonding, ${ }^{[21-23]}$ stereochemically active lone-pair electrons, ${ }^{[24-31]}$ rattling and its reminiscent anharmonic vibration, ${ }^{[30,32-42]}$ superionic transition, ${ }^{[43-47]}$ partial occupancy, ${ }^{[48,49]}$ and bonding heterogeneity ${ }^{[27,40,50-56]}$ have been unveiled. Among these, we focus on the bonding heterogeneity, namely the coexistence of strong and weak bonding, as it is more likely to be observed in many classes of crystalline solids and can be utilized more easily to realize low $\kappa_{\text {lat. }}$ The bonding heterogeneity is frequently observed in Zintl phases, which consist of coexisting ionic and covalent sublattices. In addition, local structures 
containing multiple anions should form a distorted coordination environment, which gives the bonding heterogeneity.

Mixed-anion compounds, solid-state materials containing more than one anionic species in a single phase, have recently attracted much attention because they have potential to offer novel and attractive functionalities which were not observed in conventional "single-anion" materials. ${ }^{[57]}$ Some superior properties in the mixed-anion compounds have already been discovered, such as visible-light photocatalysis, pleochroism, and battery applications. ${ }^{[57]}$ In the thermoelectrics field, anionic solid solutions like $\mathrm{PbTe}-\mathrm{PbSe}{ }^{[58,59]}$ and $\mathrm{Bi}_{2} \mathrm{Te}_{3}-\mathrm{Bi}_{2} \mathrm{Se}_{3}{ }^{[60]}$ have been regarded as an effective way to realize carrier concentration tuning, band engineering, and reduction of $\kappa_{\text {lat }}$ via phonon-alloy scattering. In addition, a mixed-anion $\mathrm{BiCuSeO}$ consisting of $\left(\mathrm{Cu}_{2} \mathrm{Se}_{2}\right)^{2-}$ and $\left(\mathrm{Bi}_{2} \mathrm{O}_{2}\right)^{2-}$ layers has been a hot material because of its extremely low $\kappa_{\text {lat }}$ and promising figure of merit. ${ }^{[61-63]}$ The origin of low $\kappa_{\text {lat }}$ is derived from large anharmonicity due to the anisotropic layered structure. It is a typical example of mixed-anion compounds containing multiple anions in separate building blocks. On the other hand, the mixed-anion are also likely to form locally distorted structures in which more than one anions are bonded to a cation. This class of materials is less explored in terms of thermal transport. Although a few previous reports ${ }^{[51-53]}$ deal with this type mixed-anion materials, unique features derived from the mixed-anions are still ambiguous because they are not compared with a single-anion counterpart. We can expect that the locally distorted structures in mixed-anion materials bring the bonding heterogeneity, which effectively realize low $\kappa_{\text {lat. }}$.

Herein, we demonstrate an effective guideline that the mixed-anion framework with distorted local structures is a promising series of materials to realize ultralow $\kappa_{\text {lat }}$. We chose Mn-based chalcohalides $\mathrm{MnPnS}_{2} \mathrm{Cl}(\mathrm{Pn}=\mathrm{Sb}, \mathrm{Bi})$ as model materials to test the validity of our strategy using mixed-anion compounds. To extract unique features derived from the mixedanion, a single-anion sulfide $\mathrm{CuTaS}_{3}$, which has some structural similarities to the mixed-anion chalcohalides, was chosen for comparison. By performing calculations based on density 
functional theory (DFT), we explore lattice dynamics and thermal transport properties of these compounds. We find a remarkable variety of second order interatomic force constants (IFC) within the distorted coordination polyhedra containing multiple anions, which shows significant bonding heterogeneity in this series. Some peak-splitting in the phonon density of states (DOS) originating from the bonding heterogeneity significantly increases the 3-phonon scattering phase space (SPS), which results in a much smaller phonon relaxation time of $\mathrm{MnPnS}_{2} \mathrm{Cl}$ than $\mathrm{CuTaS}_{3}$. Consequently, the calculated $\kappa_{\text {lat }}$ of $\mathrm{MnPnS}_{2} \mathrm{Cl}$ is strikingly about seven times lower than $\mathrm{CuTaS}_{3}$. The synthesized polycrystalline sample of $\mathrm{MnPnS}_{2} \mathrm{Cl}$ is actually demonstrated to exhibit the ultralow $\kappa_{\text {lat }}$ of about $0.5 \mathrm{~W} \mathrm{~m}^{-1} \mathrm{~K}^{-1}$ at room temperature. Considering the relatively low density and averaged atomic mass of $\mathrm{MnSbS}_{2} \mathrm{Cl}$, this mixed-anion approach can give a route to uncover lightweight materials with low $\kappa_{\text {lat. }}$.

\section{Results and Discussion}

The crystal structures of mixed-anion chalcohalides $\mathrm{MnPnCh}_{2} \mathrm{X}(\mathrm{Pn}=\mathrm{Sb}, \mathrm{Bi}, \mathrm{Ch}=\mathrm{S}, \mathrm{Se}$, $\mathrm{X}=\mathrm{Cl}, \mathrm{Br}, \mathrm{I})$ series, including our target materials, were originally investigated by singlecrystal X-ray and neutron diffraction. ${ }^{[64-66]}$ Figure 1a shows the crystal structure of the mixedanion chalcohalides visualized by VESTA 3. ${ }^{[67]} \mathrm{MnSbS}_{2} \mathrm{Cl}$ and $\mathrm{MnBiS}_{2} \mathrm{Cl}$ crystallize in the orthorhombic structure (space group Pnma). ${ }^{[64,65]}$ The primitive cell contains five crystallographically inequivalent atoms, $\mathrm{Mn}, \mathrm{Sb} / \mathrm{Bi}, \mathrm{S}(1), \mathrm{S}(2)$, and $\mathrm{Cl}$. All the atoms occupy 4c Wyckoff position. These compounds are composed of locally distorted structures derived from constituent multiple anions. The coordination environments around $\mathrm{Mn}$ and $\mathrm{Sb} / \mathrm{Bi}$ atoms are shown in Figures $1 \mathrm{~b}$ and $1 \mathrm{c}$, respectively. The $\mathrm{Mn}$ atom is octahedrally coordinated by four $\mathrm{S}$ and two $\mathrm{Cl}$ atoms with cis-geometry. The $\mathrm{Sb} / \mathrm{Bi}$ atom is coordinated by five $\mathrm{S}$ and three $\mathrm{Cl}$ atoms. The interatomic distances between $\mathrm{Sb} / \mathrm{Bi}$ and $\mathrm{Cl}$ are much longer than those between $\mathrm{Sb} / \mathrm{Bi}$ and $\mathrm{S}$ atoms. These polyhedra share their edges and vertices and form infinite chains along the $b$-axis. The locally distorted structures containing different bond lengths and chemical 
environments should result in a significant bonding heterogeneity, which in turn can cause interesting lattice dynamics and thermal transport properties.

(a)

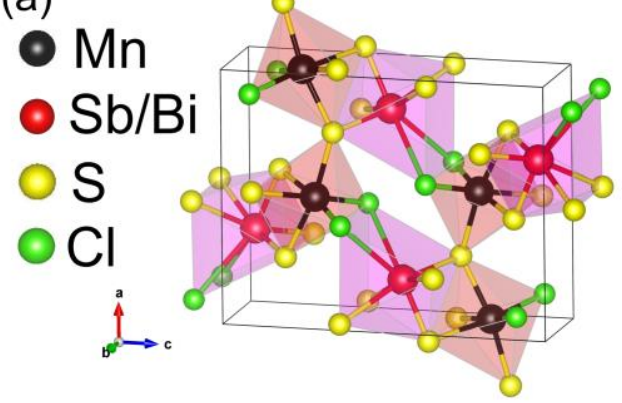

(b)

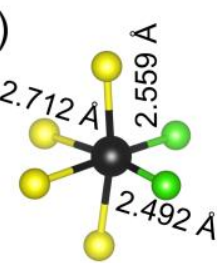

(c)

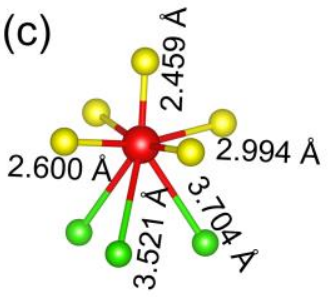

(d)

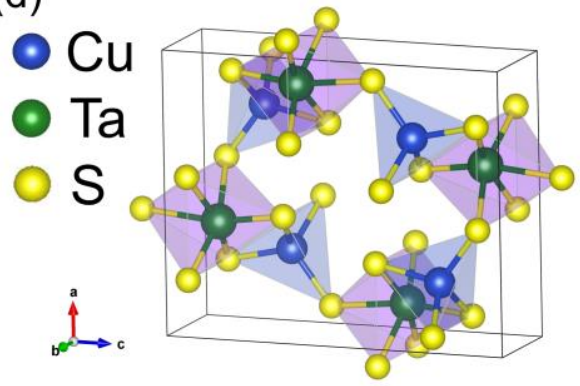

(e)

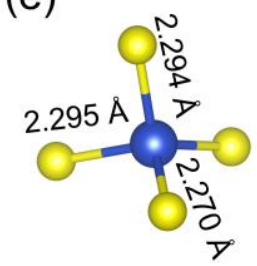

(f)

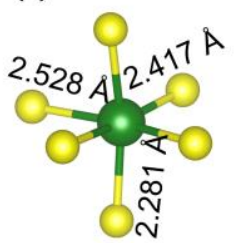

Figure 1. a) The unit cells of $\mathrm{MnPnS}_{2} \mathrm{Cl}(\mathrm{Pn}=\mathrm{Sb}, \mathrm{Bi})$ and the coordination environments around b) $\mathrm{Mn}$ and c) $\mathrm{Sb} / \mathrm{Bi}$ atoms. d) The unit cell of $\mathrm{CuTaS}_{3}$ and the coordination environment around e) $\mathrm{Cu}$ and $\mathrm{f}$ ) $\mathrm{Ta}$ atoms. The interatomic distances of atom pairs are shown along each bond. The value shown in b) and c) correspond to $\mathrm{MnSbS}_{2} \mathrm{Cl}$.

It is necessary to make a comparison between the mixed-anion compounds and a singleanion counterpart which resembles each other, in order to extract unique features originating from the mixed-anion state. $\mathrm{CuTaS}_{3}$, a single-anion sulfide, has some structural similarity to $\mathrm{MnPnS}_{2} \mathrm{Cl}$. Figure 1d shows the crystal structure of $\mathrm{CuTaS}_{3} \cdot \mathrm{CuTaS}_{3}$ crystallizes in the orthorhombic structure with space group Pnma, and the primitive cell contains five crystallographically inequivalent atoms, $\mathrm{Cu}, \mathrm{Ta}, \mathrm{S}(1), \mathrm{S}(2)$, and $\mathrm{S}(3) .{ }^{[68]}$ All the atoms occupy 4c Wyckoff position. The coordination environments around $\mathrm{Cu}$ and $\mathrm{Ta}$ atoms are shown in Figures 1e and 1f, respectively. In the ICSD database, $\mathrm{MnPnCh}_{2} \mathrm{X}$ is classified as the same structure-type as $\mathrm{CuTaS}_{3}$ though the local structures are quite different. The $\mathrm{Cu}$ atom is tetrahedrally coordinated by three $\mathrm{S}$ atoms, and the Ta atom is octahedrally coordinated by six $\mathrm{S}$ atoms. The bond lengths within the coordination polyhedra vary despite the single anion, but to a lesser extent than the mixed-anion $\mathrm{MnPnS}_{2} \mathrm{Cl}$. In addition, $\mathrm{MnBiS}_{2} \mathrm{Cl}$ and $\mathrm{CuTaS}_{3}$ have the 
similar theoretical density of $5.32 \mathrm{~g} \mathrm{~cm}^{-3}$ and $5.82 \mathrm{~g} \mathrm{~cm}^{-3}$, respectively, and the same percentage of heavy atoms where $20 \%$ of the constituent elements are heavy ( $\mathrm{Sb}, \mathrm{Bi}, \mathrm{Ta})$ and $80 \%$ are relatively light $(\mathrm{Mn}, \mathrm{Cu}, \mathrm{S}, \mathrm{Cl})$. Thus, $\mathrm{CuTaS}_{3}$ is a suitable material to compare the lattice dynamics with $\mathrm{MnPnS}_{2} \mathrm{Cl}$ to gain insight into the crucial roles of the mixed-anion.

We performed DFT-based calculations (see Computational Method in the Supporting Information) to investigate lattice dynamics and thermal transport properties of the mixed-anion $\mathrm{MnPnS}_{2} \mathrm{Cl}$ and the single-anion $\mathrm{CuTaS}_{3}$. Figure 2 shows the norm of calculated second-order IFC matrix, $\left|\Phi^{i j}\right|$ as a function of interatomic distance. The IFC represents the strength of interaction between each atomic pair, namely bond strength. The IFCs decay rapidly with increasing interatomic distance though some non-negligible components are observed for $\mathrm{Sb}$ $\mathrm{S}, \mathrm{Bi}-\mathrm{S}$, and Ta-S bonds at around $6 \AA$. The IFCs of $\mathrm{Cu}-\mathrm{S}$ bonds in the coordination tetrahedron of $\mathrm{CuTaS}_{3}$ with almost identical bond length range from 4 to $5 \mathrm{eV} \AA^{-2}$, while those of Ta-S bonds range from 4 to $7 \mathrm{eV} \AA^{-2}$ due to slightly different bond length and distorted coordination. On the other hand, the IFCs of the nearest and second nearest $\mathrm{Mn}-\mathrm{S}$ and $\mathrm{Mn}-\mathrm{Cl}$ differ by a factor of about 3-6. For the nearest Sb-S (Bi-S) and Sb-Cl (Bi-Cl), the IFCs differ by a factor of 23 (18). These indicate that the local structures in the mixed-anion $\mathrm{MnPnS}_{2} \mathrm{Cl}$ hold the remarkable bonding heterogeneity compared to $\mathrm{CuTaS}_{3}$. This significant difference of bonding environment between the mixed- and single-anion compounds can cause interesting features in lattice dynamics.
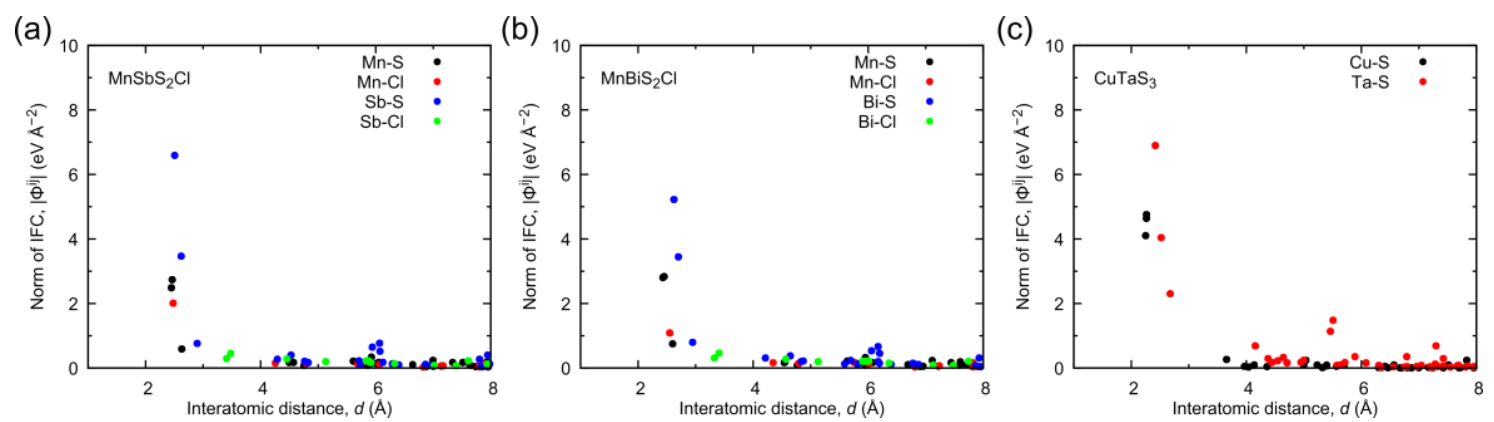

Figure 2. Norm of IFC matrix, $\left|\Phi^{\mathrm{ij}}\right|$, as a function of interatomic distance between atoms denoted by $i$ and $j$ for a) $\mathrm{MnSbS}_{2} \mathrm{Cl}$, b) $\mathrm{MnBiS}_{2} \mathrm{Cl}$, and c) $\mathrm{CuTaS}_{3}$. 
The calculated phonon dispersion curves together with the phonon DOS are shown in Figures $3 \mathrm{a}, 3 \mathrm{~b}$, and $3 \mathrm{c}$. These compounds are dynamically stable as no imaginary modes are observed in the phonon dispersion. As shown in the atom-decomposed phonon DOS, the acoustic modes mainly originate from the heaviest atom $\left(\mathrm{Sb}, \mathrm{Bi}\right.$, and $\mathrm{Ta}$ for $\mathrm{MnSbS}_{2} \mathrm{Cl}$, $\mathrm{MnBiS}_{2} \mathrm{Cl}$, and $\mathrm{CuTaS}_{3}$, respectively). For $\mathrm{CuTaS}_{3}$, high-frequency optical modes $\left(>250 \mathrm{~cm}^{-1}\right.$ ) mainly derive from $\mathrm{S}$ atom, while heavier $\mathrm{Cu}$ and $\mathrm{Ta}$ atoms mainly contribute to the lower modes $\left(<180 \mathrm{~cm}^{-1}\right)$. As a result, a gap-like opened structure is observed in the phonon DOS at around $180-250 \mathrm{~cm}^{-1}$. On the other hand, the gap-like structure is not observed in the phonon DOS of $\mathrm{MnPnS}_{2} \mathrm{Cl}$. For $\mathrm{MnPnS}_{2} \mathrm{Cl}$, high-frequency region (> $\left.200 \mathrm{~cm}^{-1}\right)$ is like $\mathrm{CuTaS}_{3}$, namely large contribution from $\mathrm{S}$ atom. However, $\mathrm{Cl}$ atom contributes to the lower frequency region $\left(70-200 \mathrm{~cm}^{-1}\right)$ despite its almost the same atomic mass as $\mathrm{S}\left(\mathrm{S}: 32.07 \mathrm{~g} \mathrm{~mol}^{-1}, \mathrm{Cl}: 35.45 \mathrm{~g}\right.$ $\mathrm{mol}^{-1}$ ). This splitting of the peak in the phonon DOS between $\mathrm{S}$ and $\mathrm{Cl}$ is derived from the bonding heterogeneity. The phonon frequency $\omega$ is related to the atomic mass and the secondorder IFC:

$\omega^{2} \propto \frac{1}{\sqrt{M_{\mathrm{A}} M_{\mathrm{B}}}} \sum \Phi$

where $M_{\mathrm{A}}$ and $M_{\mathrm{B}}$ are the atomic mass of species $\mathrm{A}$ and $\mathrm{B}$, respectively, and $\Phi$ is the secondorder IFC. Thus, a significant variety of the IFC originating from the bonding heterogeneity results in the splitting of phonon frequency, even though the atomic mass of multiple anions is almost the same. Figure $3 \mathrm{~d}$ shows a schematic diagram representing the situation occurred here. The gap-like structure observed in the phonon DOS of the single-anion material is filled with additional states generated from the peak splitting induced by the bonding heterogeneity in the mixed-anion material with locally distorted structures. 

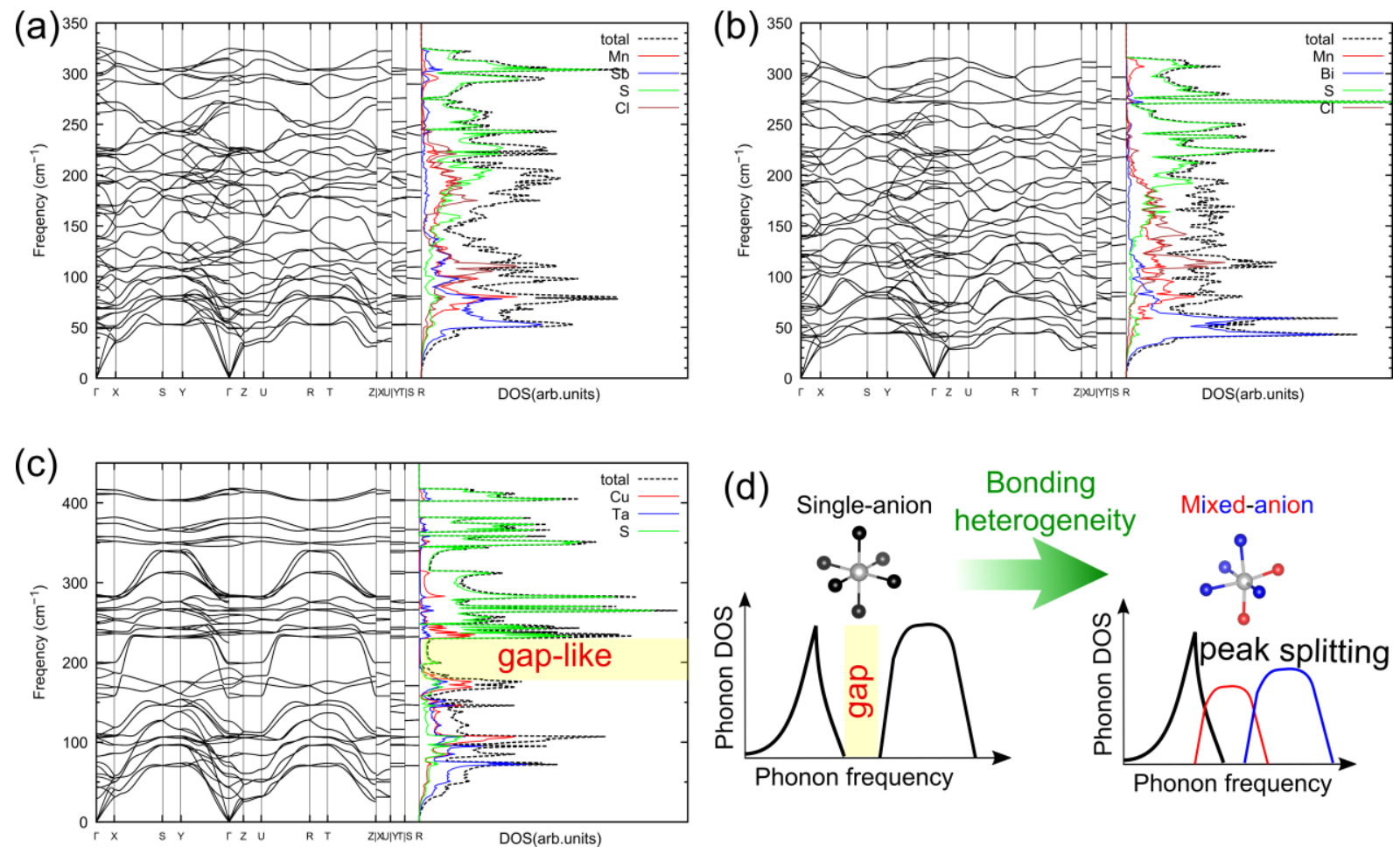

Figure 3. The calculated phonon dispersion and the total and atom-decomposed phonon DOS for a) $\mathrm{MnSbS}_{2} \mathrm{Cl}$, b) $\mathrm{MnBiS}_{2} \mathrm{Cl}$, and c) $\mathrm{CuTaS}_{3}$. A colored region in c) represents a gap-like opened structure observed in the phonon DOS of $\mathrm{CuTaS}_{3}$. d) A schematic diagram representing the change of phonon DOS from single-anion to mixed-anion materials induced by the bonding heterogeneity. The gap-like structure of DOS observed in the single-anion is filled with additional states formed by peak splitting in the mixed-anion one.

A potential energy surface that provides us with insight into anharmonicity at each atomic site can be visualized by plotting an energy change with respect to a displacement of the atom. Figures $4 \mathrm{a}, 4 \mathrm{~b}$, and $4 \mathrm{c}$ show the potential energy of anion sites as a function of displacements along cartesian $x$-, $y$-, and $z$-directions for $\mathrm{MnSbS}_{2} \mathrm{Cl}, \mathrm{MnBiS}_{2} \mathrm{Cl}$, and $\mathrm{CuTaS}_{3}$, respectively. The potential energy of all sites, including cation sites, is shown in Figure S1 in the Supporting Information. The potential energy exhibits slightly asymmetric features and deviates from parabolic behavior, which shows the high anharmonicity of these compounds. The $\mathrm{Cl}$ atoms of $\mathrm{MnPnS}_{2} \mathrm{Cl}$ lie in a flatter potential than the $\mathrm{S}$ atoms, which is consistent with the weaker bonding environment and the lower phonon frequency of the $\mathrm{Cl}$ atoms. The shallow potential well also corresponds to the large thermal displacement parameters represented by the mean square displacement, as shown in Figure S2 in the Supporting Information. To further gain insight into 
the anharmonicity of these compounds, we calculated the Grüneisen parameters as shown in Figure $4 \mathrm{~d}$. The Grüneisen parameter is defined by the change of phonon frequency with respect to the change of volume of the unit cell. Some compounds possessing lone-pair electrons and low $\kappa_{\text {lat }}$ show anomalously large Grüneisen parameter exceeding $50 .{ }^{[24,27]}$ For the low frequency region of acoustic modes, the magnitude of the Grüneisen parameter is larger for $\mathrm{CuTaS}_{3}$ than $\mathrm{MnPnS}_{2} \mathrm{Cl}$ while $\mathrm{MnPnS}_{2} \mathrm{Cl}$ has larger values at around $50 \mathrm{~cm}^{-1}$ and $150 \mathrm{~cm}^{-1}$. From the Grüneisen parameter, we can see that both $\mathrm{MnPnS}_{2} \mathrm{Cl}$ and $\mathrm{CuTaS}_{3}$ has moderate anharmonicity compared to extremely anharmonic materials. ${ }^{[24,27]}$
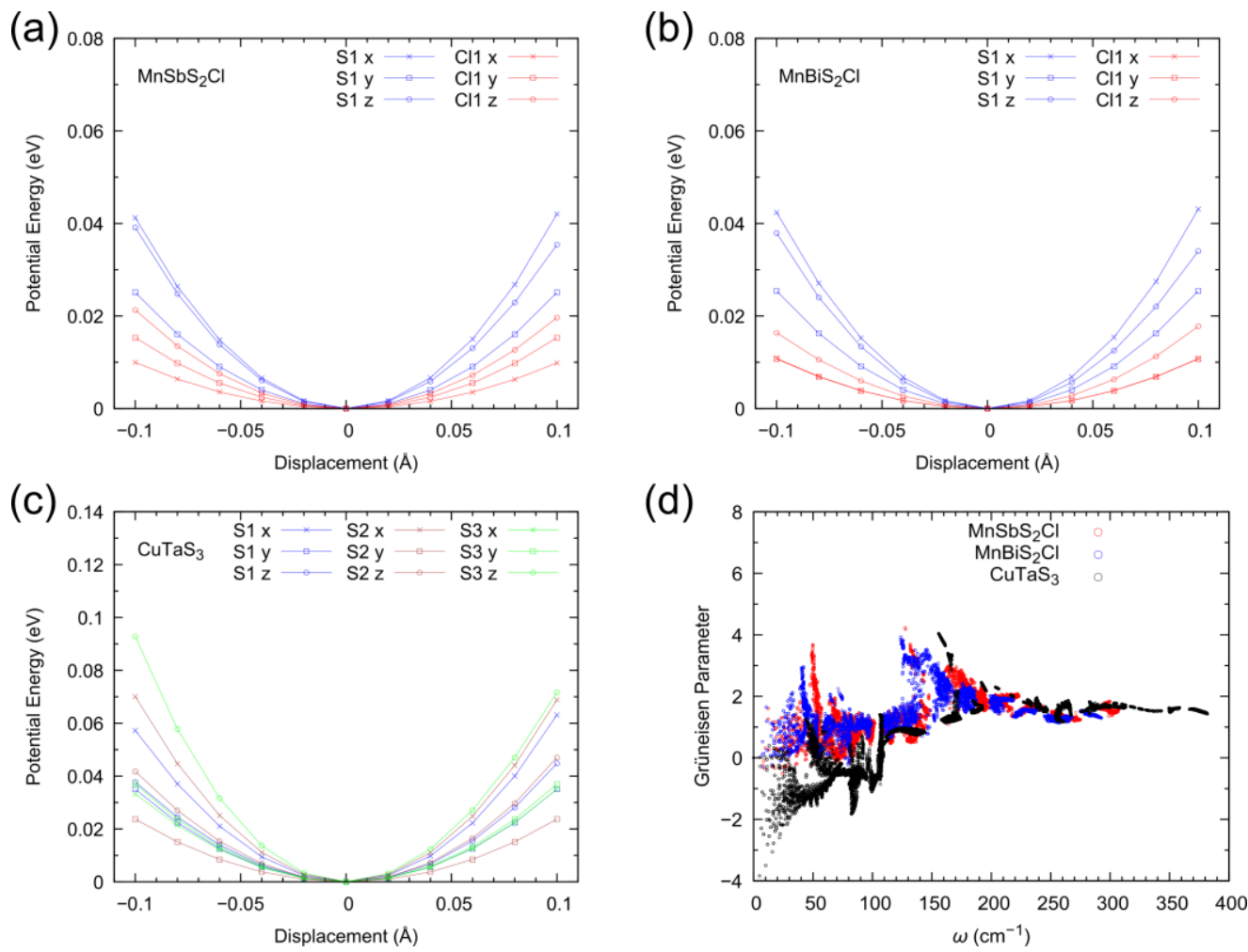

Figure 4. The calculated potential energy of each atomic site as a function of displacements along cartesian $x$-, $y$-, and $z$-directions for a) $\mathrm{MnSbS}_{2} \mathrm{Cl}$, b) $\mathrm{MnBiS}_{2} \mathrm{Cl}$, and c) $\mathrm{CuTaS}_{3}$. d) The calculated Grüneisen parameter as a function of the phonon frequency.

To achieve further insight into the relationship between the bonding heterogeneity and the thermal transport, we calculated the 3-phonon SPS. The SPS is a measure of the amount of available scattering processes satisfying the energy and momentum conservation. The SPS is given as 
$W_{q}^{ \pm}=\frac{1}{N} \sum_{q^{\prime}, q^{\prime \prime}}\left\{\begin{array}{c}n_{q^{\prime \prime}}-n_{q^{\prime}} \\ n_{q^{\prime}}+n_{q^{\prime \prime}}+1\end{array}\right\} \delta\left(\omega_{q}-\omega_{q^{\prime}} \pm \omega_{q^{\prime \prime}}\right)$

where $W_{q}^{+}$and $W_{q}^{-}$are the SPS corresponding to absorption and emission processes of phonon mode $q$, respectively. Here, the variable $q$ is defined by $q=(\boldsymbol{q}, j)$ and $-q=(-\boldsymbol{q}, j)$ where $\boldsymbol{q}$ and $j$ are the wave vector and the branch index of phonon modes. $n_{q}$ is the Bose-Einstein distribution function. Figures $5 \mathrm{a}$ and $5 \mathrm{~b}$ show $W_{q}^{+}$and $W_{q}^{-}$as a function of the phonon freuquency at $300 \mathrm{~K}$, respectively. Notably, $W_{q}^{+}$at the low-frequency region and $W_{q}^{-}$at the middle and high-frequency region are several times larger for $\mathrm{MnPnS}_{2} \mathrm{Cl}$ than $\mathrm{CuTaS}_{3}$. These differences should originate from the peak splitting of phonon DOS discussed above, as represented by Figure 3d. The gap-like structure observed in the phonon DOS causes the lower amount of 3-phonon scattering processes between acoustic-optical and optical-optical modes. The gap is filled with the additional states induced by the bonding heterogeneity, which results in the increase of acoustic-optical and optical-optical scattering processes. The SPS is proportional to the 3-phonon scattering rate, the inverse of phonon relaxation time $\tau$. In other words, the larger the SPS, the smaller the $\tau$. Thus, the larger SPS brings the lower $\kappa_{\text {lat. }}$ Figure $5 \mathrm{c}$ shows the 3-phonon relaxation time as a function of the phonon frequency at $300 \mathrm{~K}$. At the overall frequency range, the $\tau$ of $\mathrm{MnPnS}_{2} \mathrm{Cl}$ is significantly smaller than that of $\mathrm{CuTaS}_{3}$. As shown in Figure S3 in the Supporting Information, the phonon group velocity of acoustic modes of $\mathrm{MnPnS}_{2} \mathrm{Cl}$ and $\mathrm{CuTaS}_{3}$ is not essentially different, while the optical modes of $\mathrm{CuTaS}_{3}$ at around $150-250 \mathrm{~cm}^{-1}$, corresponding to the dispersive curve in Figure $3 \mathrm{c}$, have much larger value. However, this large group velocity of the optical modes of $\mathrm{CuTaS}_{3}$ has a negligible contribution to the lattice thermal conductivity, as shown in the spectral thermal conductivity (Figure S4, Supporting Information). Therefore, the significant difference in the $\tau$ between $\mathrm{MnPnS}_{2} \mathrm{Cl}$ and $\mathrm{CuTaS}_{3}$ is a dominant factor determining the difference in $\kappa_{\text {lat. }}$ 


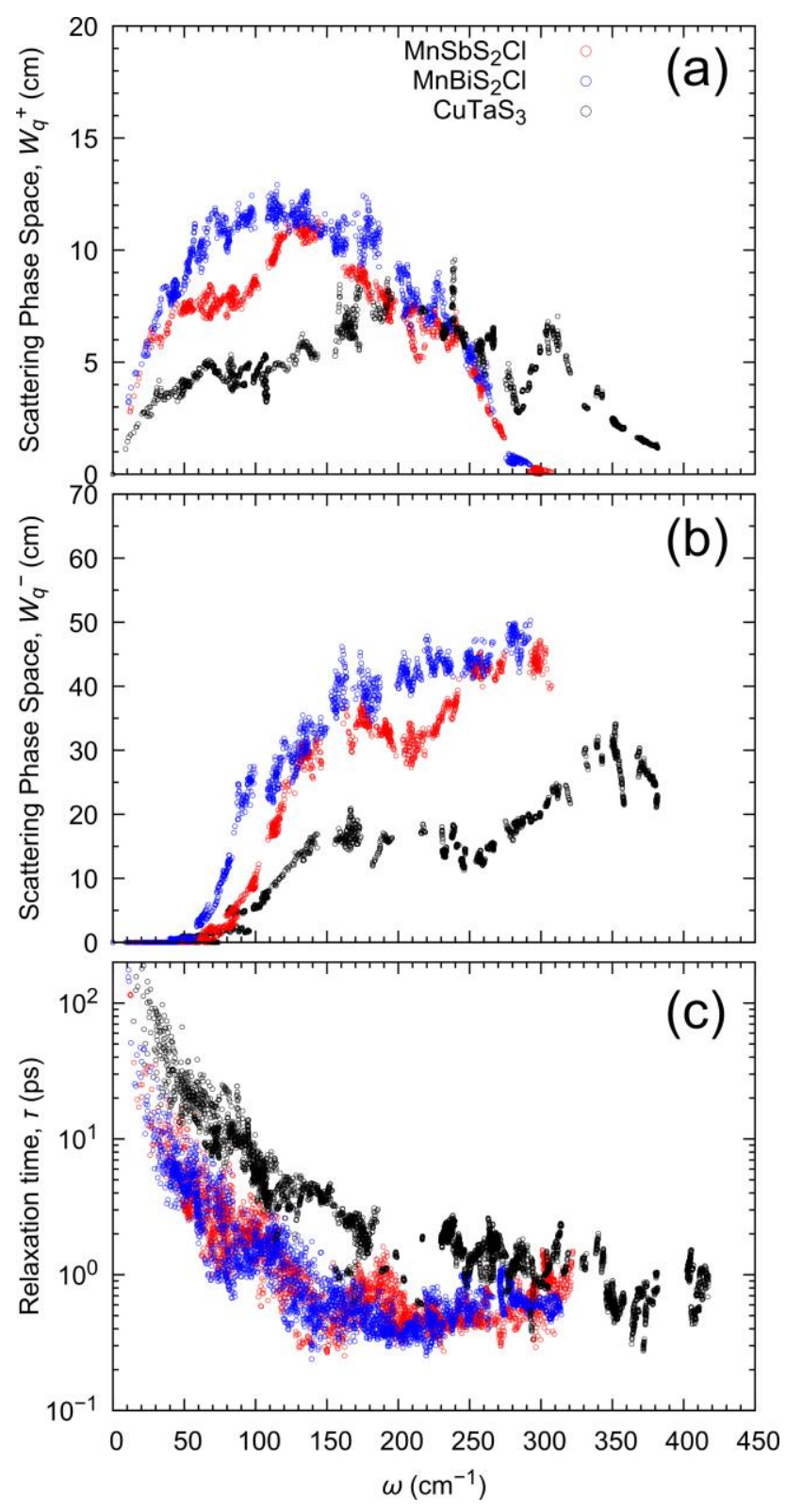

Figure 5. The calculated 3-phonon SPS for a) absorption and b) emission processes, and c) 3phonon relaxation time at $300 \mathrm{~K}$ as a function of the phonon frequency.

We calculated the $\kappa_{\text {lat }}$ by solving the phonon Boltzmann transport equation within the single mode relaxation time approximation. The calculation only considers the intrinsic 3-phonon scattering. Figures $6 \mathrm{a}, 6 \mathrm{~b}$, and $6 \mathrm{c}$ show the temperature dependence of the $\kappa_{\text {lat }}(x x, y y$, and $z z$ components) for $\mathrm{MnSbS}_{2} \mathrm{Cl}, \mathrm{MnBiS}_{2} \mathrm{Cl}$, and $\mathrm{CuTaS}_{3}$, respectively. The averaged value of each direction is also shown. In addition, we synthesized the polycrystalline samples of $\mathrm{MnSbS}_{2} \mathrm{Cl}$, $\mathrm{MnBiS}_{2} \mathrm{Cl}$, and $\mathrm{CuTaS}_{3}$ by solid-state reaction, melting, and spark plasma sintering and measured the experimental $\kappa_{\text {lat }}$ (see Experimental Procedure and measured X-ray diffraction 
patterns in Figure S5 in the Supporting Information). The temperature dependence of the experimental $\kappa_{\text {lat }}$ is plotted in Figures $6 \mathrm{a}, 6 \mathrm{~b}$, and $6 \mathrm{c}$. The calculated $\kappa_{\text {lat }}$ shows a significant anisotropy for these compounds due to the anisotropic crystal structures. The averaged calculated values at $300 \mathrm{~K}$ are $1.1 \mathrm{~W} \mathrm{~m}^{-1} \mathrm{~K}^{-1}, 1.0 \mathrm{~W} \mathrm{~m}^{-1} \mathrm{~K}^{-1}$, and $7.2 \mathrm{~W} \mathrm{~m}^{-1} \mathrm{~K}^{-1}$ for $\mathrm{MnSbS}_{2} \mathrm{Cl}$, $\mathrm{MnBiS}_{2} \mathrm{Cl}$, and $\mathrm{CuTaS}_{3}$, respectively. The temperature dependence of the experimental $\kappa_{\text {lat }}$ for $\mathrm{MnPnS}_{2} \mathrm{Cl}$ is flat, while the calculated one obeys $T^{-1}$ dependence. The experimental values at $300 \mathrm{~K}$ are $0.50 \mathrm{~W} \mathrm{~m}^{-1} \mathrm{~K}^{-1}, 0.53 \mathrm{~W} \mathrm{~m}^{-1} \mathrm{~K}^{-1}$, and $3.5 \mathrm{~W} \mathrm{~m}^{-1} \mathrm{~K}^{-1}$, respectively. Both the calculated and experimental values differ strikingly by a factor of 7 between $\mathrm{MnPnS}_{2} \mathrm{Cl}$ and $\mathrm{CuTaS}_{3}$. The deviation between the absolute values of calculated and experimental $\kappa_{\text {lat }}$ can be due to several factors: the experimental $\kappa_{\text {lat }}$ contains the effect of grain boundary and chemical disorder. Moreover, some effects unveiled in recent years are beyond the scope of our calculations, such as the temperature dependent anharmonicity ${ }^{[6]}$ and crystal/amorphous boundary behavior, ${ }^{[70,71]}$ which might contribute to the glass-like flat temperature dependence.

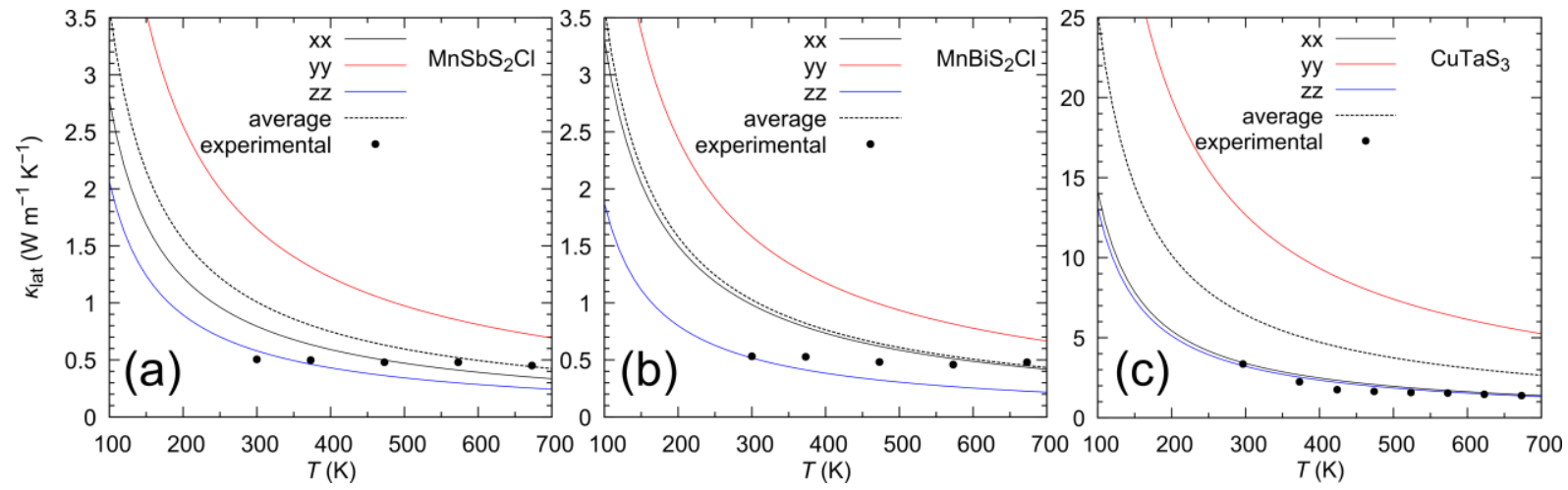

Figure 6. The temperature dependence of calculated and experimental $\kappa_{\text {lat }}$ for a) $\mathrm{MnSbS}_{2} \mathrm{Cl}$, b) $\mathrm{MnBiS}_{2} \mathrm{Cl}$, and c) $\mathrm{CuTaS}_{3}$.

We have discovered that the mixed-anion $\mathrm{MnPnS}_{2} \mathrm{Cl}$ exhibits the ultralow $\kappa_{\text {lat }}$ even at room temperature. Considering the relatively low density $\left(\mathrm{MnSbS}_{2} \mathrm{Cl}: 4.12 \mathrm{~g} \mathrm{~cm}^{-3}, \mathrm{MnBiS}_{2} \mathrm{Cl}: 5.32\right.$ $\left.\mathrm{g} \mathrm{cm}^{-3}\right)$ and averaged atomic mass per atom $\left(\mathrm{MnSbS}_{2} \mathrm{Cl}: 55.27 \mathrm{~g} \mathrm{~mol}^{-1}\right.$ atom $^{-1}, \mathrm{MnBiS}_{2} \mathrm{Cl}$ : $72.71 \mathrm{~g} \mathrm{~mol}^{-1}$ atom $^{-1}$ ), the $\kappa_{\text {lat }}$ values of these compounds are unexpectedly low. Light atoms generally make stronger and more covalent bonding than heavier ones, which typically leads to 
high $\kappa_{\text {lat }}$. To highlight the anomaly of the mixed-anion compounds, we plotted the $\kappa_{\text {lat }}$ versus the averaged atomic mass of various materials, including simple semiconductors and some known thermoelectric materials with low $\kappa_{\text {lat }}$ in Figure 7 . Almost all materials with $\kappa_{\text {lat }}<1 \mathrm{~W}$ $\mathrm{m}^{-1} \mathrm{~K}^{-1}$ shown here have large averaged atomic mass larger than $80 \mathrm{~g} \mathrm{~mol}^{-1}$ atom ${ }^{-1}$, except for $\mathrm{MnPnS}_{2} \mathrm{Cl}, \mathrm{Cu}_{2} \mathrm{~S},{ }^{[47]}$ and $\mathrm{Cu}_{4} \mathrm{TiSe}_{4}{ }^{[72]} \mathrm{Cu}_{2} \mathrm{~S}$ undergoes two phase transitions and possesses liquid-like copper ions, which results in ultralow $\kappa_{\text {lat. }}{ }^{[47]}$ The extremely low $\kappa_{\text {lat }}$ of $\mathrm{Cu}_{4} \mathrm{TiSe}_{4}$, $0.19 \mathrm{~W} \mathrm{~m}^{-1} \mathrm{~K}^{-1}$ at $300 \mathrm{~K}$, was very recently confirmed and attributed to its highly disordered structure and soft phonon modes. ${ }^{[72]}$ Note that the lightweight and ultralow $\kappa_{\text {lat }} \mathrm{MnPnS} \mathrm{Sl}_{2}$ contains none of the phase transitions, structural instability, and extremely high anharmonicity but contains the bonding heterogeneity induced by the locally distorted structures.

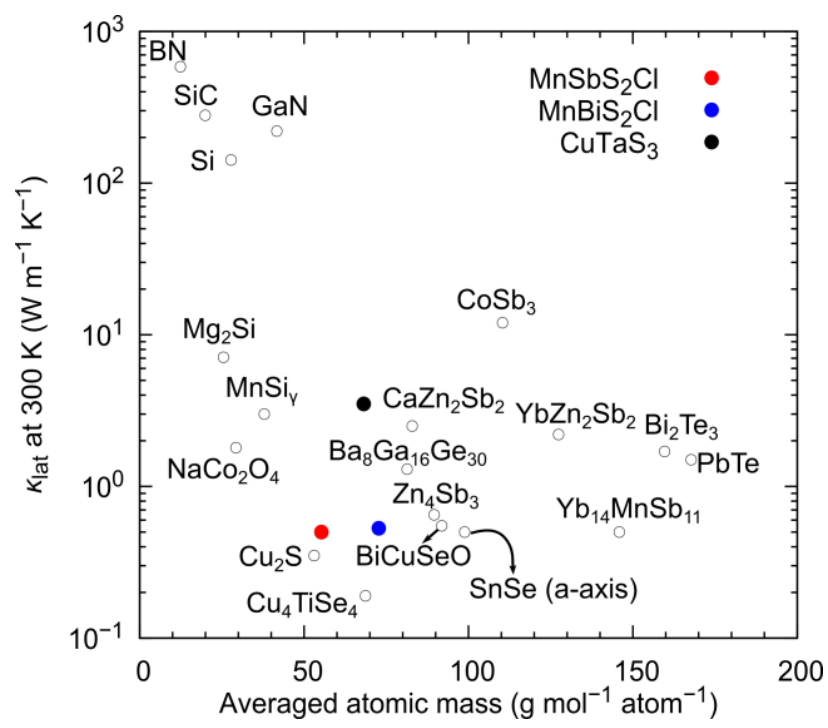

Figure 6. $\kappa_{\text {lat }}$ at $300 \mathrm{~K}$ versus averaged atomic mass for various compounds including the mixed-anion $\mathrm{MnPnS}_{2} \mathrm{Cl}$ (this work), $\mathrm{CuTaS}_{3}$ (this work), h-BN (in-plane), ${ }^{[73]} 4 \mathrm{H}-\mathrm{SiC},{ }^{[74]}$

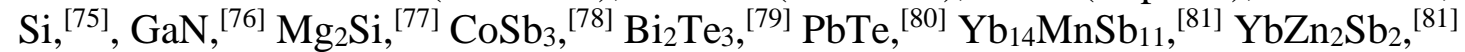
$\mathrm{CaZn}_{2} \mathrm{Sb}_{2},{ }^{[81]} \mathrm{Zn}_{4} \mathrm{Sb}_{3},{ }^{[82]} \mathrm{MnSi}_{\gamma},{ }^{[14]} \mathrm{Cu}_{2} \mathrm{~S},{ }^{[47]} \mathrm{Ba}_{8} \mathrm{Ga}_{16} \mathrm{Ge}_{30},{ }^{[36]} \mathrm{BiCuSeO},{ }^{[61]} \mathrm{SnSe}\left(a\right.$-axis), ${ }^{[83]}$ $\mathrm{NaCO}_{2} \mathrm{O}_{4},{ }^{[84]}$ and $\mathrm{Cu}_{4} \mathrm{TiSe}_{4}{ }^{[72]}$

A great advantage of the bonding heterogeneity by mixed-anion concept to realize low $\kappa_{\text {lat }}$, summarized in Figure 3d, is that the phonon frequency of light atoms, like $\mathrm{Cl}$ in this work, can be lowered so that the number of optical modes whose frequency are close to acoustic modes increases. It means that the strategy can be utilized to explore lightweight and low $\kappa_{\text {lat }}$ materials. 
In particular, abundant mixed-anion materials unknown in terms of thermal conductivity, such as oxynitrides, oxysulfides, and chalcohalides, can be promising candidates.

\section{Conclusion}

In conclusion, we have demonstrated that the locally distorted structures observed in the mixed-anion compounds derive ultralow $\kappa_{\text {lat. }}$ Using DFT-based calculations, we clarified the significant variety of the second-order IFC in the mixed-anion $\mathrm{MnPnS}_{2} \mathrm{Cl}$, which represents the bonding heterogeneity. This bonding environment causes the remarkable splitting of the phonon frequency, which essentially increases the amount of 3-phonon scattering processes. The increased SPS leads to the lower phonon relaxation time. Consequently, $\kappa_{\text {lat }}$ of $\mathrm{MnPnS}_{2} \mathrm{Cl}$ is significantly lower than that of a single-anion sulfide $\mathrm{CuTaS}_{3}$ with the similar crystal structure and percentage of heavy atoms. Experimental $\kappa_{\text {lat }}$ of $\mathrm{MnPnS}_{2} \mathrm{Cl}$ shows an ultralow value of about $0.5 \mathrm{~W} \mathrm{~m}^{-1} \mathrm{~K}^{-1}$ at room temperature, which is unexpectedly low considering the relatively low averaged atomic mass per atom of these compounds. A possible future work will be to confirm whether the strategy found in this work can be applied to a broader range of the mixedanion compounds. Our study decorates the mixed-anion compounds, which remain a vast unexplored space, with novel thermal functionality.

\section{Experimental Section}

Synthesis: Polycrystalline samples of $\mathrm{MnPnS}_{2} \mathrm{Cl}$ were synthesized by solid state reaction of stoichiometric amounts of $\mathrm{MnS}, \mathrm{MnCl}_{2}$, and $\mathrm{Pn}_{2} \mathrm{~S}_{3}$ (1:1:1). The starting materials were weighed and ground with an agate mortar in an Ar-filled glovebox, and then transferred to quartz tubes and sealed under a high vacuum. These ampoules were put into a box furnace and heated to 773 $\mathrm{K}$ for 2 hours, and kept for 7 days. The obtained ingots were ground into fine powders $(<45$ $\mu \mathrm{m}$ ) and consolidated by spark plasma sintering (SPS, SPS-515S, Fuji Electronic Industrial Co., Japan). To obtain dense and high-purity bulk samples, we applied high-pressure and lowtemperature conditions for SPS. The powder samples were set in a $10 \mathrm{~mm}$ diameter cemented 
carbide die, heated to $813 \mathrm{~K}$ with a uniaxial pressure of $500 \mathrm{MPa}$ under Ar atmosphere, and then held for $30 \mathrm{~min}$. The dense bulk samples with relative density $\sim 98 \%$ were obtained.

A polycrystalline sample of $\mathrm{CuTaS}_{3}$ was synthesized by melting and following solid state reaction of element powders $\mathrm{Cu}$, Ta, and $\mathrm{S}$. To avoid S-poor impurity phases, the nominal composition was set to $\mathrm{CuTaS}_{3.2}$. The starting materials were weighed and ground with the agate mortar, and then transferred to a quartz tube and sealed under a high vacuum. The ampoule was put into the box furnace and heated to $923 \mathrm{~K}$ for 24 hours, and kept for 7 days. The obtained ingot was ground into fine powders $(<45 \mu \mathrm{m})$ and consolidated by SPS. The powder sample was set in a $10 \mathrm{~mm}$ diameter graphite die, heated to $873 \mathrm{~K}$ with a uniaxial pressure of $100 \mathrm{MPa}$ under Ar atmosphere, and then held for $10 \mathrm{~min}$. The dense bulk sample with relative density $\sim 98 \%$ was obtained.

Characterization: The phase identification of the samples was performed by X-ray diffraction (XRD) measurements using $\mathrm{Cu} \mathrm{K}_{\alpha}$ radiation (SmartLab, Rigaku Co., Japan). The thermal conductivity $\kappa$ was brought by $\kappa=D C_{\mathrm{p}} d$, where $D, C_{\mathrm{p}}$, and $d$ are the thermal diffusivity, the specific heat, and the density, respectively. The thermal diffusivity and the specific heat were concurrently measured for the disk-shaped samples by laser flash method (LFA 467 Hyperflash, Netzsch, Germany) with a pyroceram disk as a reference sample. The sample density was measured by Archimedes method. The lattice thermal conductivity $\kappa_{\text {lat }}$ can be regarded as the same as the measured $\kappa$ because $\mathrm{MnPnS}_{2} \mathrm{Cl}$ and $\mathrm{CuTaS}_{3}$ are insulators with high electrical resistivity.

Computational Method: We performed ab initio calculations based on density functional theory (DFT). The Quantum Espresso (QE) package ${ }^{[85,86]}$ with projector-augmented wave pseudopotentials $^{[87,88]}$ was used for total energy calculations. The generalized gradient approximation functional with Perdew-Burke-Ernzerhof parametrization revised for solids (GGA-PBEsol) $^{[89]}$ was employed for the exchange and correlation potentials. For $\mathrm{MnPnS}_{2} \mathrm{Cl}$ $(\mathrm{Pn}=\mathrm{Sb}, \mathrm{Bi})$, we employed spin-polarized settings with oppositely oriented magnetic moments 
on Mn atoms. Initial values of magnetic moments of Mn atoms were set to $\pm 5 \mu_{\mathrm{B}}$, and after structure relaxation, they were converged to $\pm 4.19 \mu_{\mathrm{B}}$ and $\pm 4.15 \mu_{\mathrm{B}}$ for $\mathrm{MnSbS}_{2} \mathrm{Cl}$ and $\mathrm{MnBiS}_{2} \mathrm{Cl}$, respectively. $k$-point grids of $6 \times 14 \times 4$ and $6 \times 15 \times 4$ and energy cutoff of $100 \mathrm{Ry}$ and $90 \mathrm{Ry}$ for the primitive cell of $\mathrm{MnPnS}_{2} \mathrm{Cl}$ and $\mathrm{CuTaS}_{3}$, respectively, were used for structure relaxation. The lattice parameters of the relaxed primitive cell of $\mathrm{MnPnS}_{2} \mathrm{Cl}$ and $\mathrm{CuTaS}_{3}$ are listed in Table S1.

To extract the second and third order interatomic force constants (IFC), we used finite difference method with $2 \times 3 \times 1$ supercells containing 120 atoms based on the fully relaxed primitive cell to create displacement-force datasets. The magnitude of atomic displacements was set at $0.01 \AA$ and $0.04 \AA$ for calculating the second and third order IFCs, respectively. For the third order IFC calculation, we chose the cutoff radius as $14.2 \AA$ for choosing triplets within which interaction is considered. The IFCs were obtained using ALAMODE ${ }^{[90]}$ code. The phonon dispersion relation and density of states (DOS) were calculated from the second order IFC. The sampling grids used for summation were $7 \times 17 \times 5$ and $7 \times 18 \times 6$ for $\mathrm{MnPnS}_{2} \mathrm{Cl}$ and $\mathrm{CuTaS}_{3}$, respectively. Dielectric constant and Born effective charge were calculated in the perturbation framework implemented in the QE package for correcting phonon frequencies considering long-range interaction. ALAMODE code is also used to calculate the lattice thermal conductivity by solving the phonon Boltzmann transport equation within the single mode relaxation time approximation. The sampling grids $7 \times 17 \times 5$ and $7 \times 18 \times 6$ for $\mathrm{MnSbS}_{2} \mathrm{Cl}$ and $\mathrm{MnBiS}_{2} \mathrm{Cl}$, respectively, were applied after confirming convergence of the calculated lattice thermal conductivity with respect to the grid density.

\section{Acknowledgements}

This work was supported by the JSPS JP16H06441, JP17H02749, and JST Mirai Program JPMJMI19A1, CREST JPMJCR19Q4. The computation in this work has been performed using the Numerical Materials Simulator at NIMS and SGI Rackable C2112-4GP3/C1102GP8 (Reedbush-U/H/L) in the Information Technology Center, The University of Tokyo. 
References

[1] J. Mao, Z. Liu, J. Zhou, H. Zhu, Q. Zhang, G. Chen, Z. Ren, Adv. Phys. 2018, 67, 69.

[2] T. Mori, Small 2017, 13, 1702013.

[3] N. Nandihalli, C.-J. Liu, T. Mori, Nano Energy 2020, 78, 105186.

[4] R. Vaßen, M. O. Jarligo, T. Steinke, D. E. Mack, D. Stöver, Surf. Coatings Technol. 2010, 205, 938.

[5] D. M. Smith, A. Maskara, U. Boes, J. Non. Cryst. Solids 1998, 225, 254.

[6] L. Hu, T. Zhu, X. Liu, X. Zhao, Adv. Funct. Mater. 2014, 24, 5211.

[7] Y. Cao, X. Su, F. Meng, T. P. Bailey, J. Zhao, H. Xie, J. He, C. Uher, X. Tang, Adv. Funct. Mater. 2020, 30, 2005861.

[8] B. Poudel, Q. Hao, Y. Ma, Y. Lan, A. Minnich, B. Yu, X. Yan, D. Wang, A. Muto, D. Vashaee, X. Chen, J. Liu, M. S. Dresselhaus, G. Chen, Z. Ren, Science (80-. ). 2008, 320, 634.

[9] S. H. Lo, J. He, K. Biswas, M. G. Kanatzidis, V. P. Dravid, Adv. Funct. Mater. 2012, $22,5175$.

[10] K. Biswas, J. He, I. D. Blum, C.-I. Wu, T. P. Hogan, D. N. Seidman, V. P. Dravid, M. G. Kanatzidis, Nature 2012, 489, 414.

[11] N. Sato, H. Ouchi, Y. Takagiwa, K. Kimura, Chem. Mater. 2016, $28,529$.

[12] Y. Iwasaki, K. Kitahara, K. Kimura, Phys. Rev. Mater. 2019, 3, 061601.

[13] Y. Miyazaki, D. Igarashi, K. Hayashi, T. Kajitani, K. Yubuta, Phys. Rev. B 2008, 78, 214104.

[14] Y. Miyazaki, H. Hamada, H. Nagai, K. Hayashi, Materials (Basel). 2018, 11, 926.

[15] T. Takeuchi, Zeitschrift für Krist. - Cryst. Mater. 2009, 224, 35.

[16] K. Tobita, N. Sato, K. Kitahara, Y. Takagiwa, K. Kimura, Mater. Trans. 2016, 57, 1045.

[17] W. Li, J. Carrete, G. K. H. Madsen, N. Mingo, Phys. Rev. B 2016, 93, 205203. 
[18] G. A. Slack, In Semiconductors and Semimetals (Eds.: Seitz, F.; Turnbull, D.;

Ehrenreich, H.), Academic Press, New York, 1979, p. 1.

[19] T. Mori, J. Solid State Chem. 2019, 275, 70.

[20] Y. Kakefuda, K. Yubuta, T. Shishido, A. Yoshikawa, S. Okada, H. Ogino, N.

Kawamoto, T. Baba, T. Mori, APL Mater. 2017, 5, 126103.

[21] S. Lee, K. Esfarjani, T. Luo, J. Zhou, Z. Tian, G. Chen, Nat. Commun. 2014, 5, 3525.

[22] S. Maier, S. Ohno, G. Yu, S. D. Kang, T. C. Chasapis, V. A. Ha, S. A. Miller, D.

Berthebaud, M. G. Kanatzidis, G.-M. Rignanese, G. Hautier, G. J. Snyder, F. Gascoin, Chem. Mater. 2018, 30, 174.

[23] W. Zhang, N. Sato, K. Tobita, K. Kimura, T. Mori, Chem. Mater. 2020, 32, 5335.

[24] M. D. Nielsen, V. Ozolins, J. P. Heremans, Energy Environ. Sci. 2013, 6, 570.

[25] Y. Dong, A. R. Khabibullin, K. Wei, J. R. Salvador, G. S. Nolas, L. M. Woods, ChemPhysChem 2015, 16, 3264.

[26] M. Dutta, K. Pal, U. V. Waghmare, K. Biswas, Chem. Sci. 2019, 10, 4905.

[27] K. Pal, J. He, C. Wolverton, Chem. Mater. 2018, 30, 7760.

[28] M. Dutta, M. Samanta, T. Ghosh, D. J. Voneshen, K. Biswas, Angew. Chemie Int. Ed. 2021, 60, 4259.

[29] P. Acharyya, T. Ghosh, K. Pal, K. Kundu, K. Singh Rana, J. Pandey, A. Soni, U. V. Waghmare, K. Biswas, J. Am. Chem. Soc. 2020, 142, 15595.

[30] M. K. Jana, K. Pal, U. V. Waghmare, K. Biswas, Angew. Chemie Int. Ed. 2016, 55, 7792.

[31] B. Du, K. Chen, H. Yan, M. J. Reece, Scr. Mater. 2016, 111, 49.

[32] G. S. Nolas, D. T. Morelli, T. M. Tritt, Annu. Rev. Mater. Sci. 1999, 29, 89.

[33] Y. Zhu, Z. Han, F. Jiang, E. Dong, B.-P. Zhang, W. Zhang, W. Liu, Mater. Today Phys. 2021, 16, 100327. 
[34] S. O. Long, A. V. Powell, S. Hull, F. Orlandi, C. C. Tang, A. R. Supka, M. Fornari, P. Vaqueiro, Adv. Funct. Mater. 2020, 30, 1909409.

[35] Z. Liu, N. Sato, W. Gao, K. Yubuta, N. Kawamoto, M. Mitome, K. Kurashima, Y.

Owada, K. Nagase, C.-H. Lee, J. Yi, K. Tsuchiya, T. Mori, Joule 2021, 5, 1196.

[36] T. Takabatake, K. Suekuni, T. Nakayama, E. Kaneshita, Rev. Mod. Phys. 2014, 86, 669.

[37] K. Suekuni, C. H. Lee, H. I. Tanaka, E. Nishibori, A. Nakamura, H. Kasai, H. Mori, H. Usui, M. Ochi, T. Hasegawa, M. Nakamura, S. Ohira-Kawamura, T. Kikuchi, K. Kaneko, H. Nishiate, K. Hashikuni, Y. Kosaka, K. Kuroki, T. Takabatake, Adv. Mater. 2018, 30, 1706230.

[38] M. K. Jana, K. Pal, A. Warankar, P. Mandal, U. V Waghmare, K. Biswas, J. Am. Chem. Soc. 2017, 139, 4350.

[39] M. Dutta, S. Matteppanavar, M. V. D. Prasad, J. Pandey, A. Warankar, P. Mandal, A. Soni, U. V. Waghmare, K. Biswas, J. Am. Chem. Soc. 2019, 141, 20293.

[40] K. Pal, Y. Xia, J. He, C. Wolverton, Phys. Rev. Mater. 2019, 3, 085402.

[41] H. Lin, G. Tan, J.-N. Shen, S. Hao, L.-M. Wu, N. Calta, C. Malliakas, S. Wang, C.

Uher, C. Wolverton, M. G. Kanatzidis, Angew. Chemie Int. Ed. 2016, 55, 11431.

[42] K. Pal, Y. Xia, J. He, C. Wolverton, Chem. Mater. 2019, 31, 8734.

[43] H. Liu, X. Shi, F. Xu, L. Zhang, W. Zhang, L. Chen, Q. Li, C. Uher, T. Day, G. J. Snyder, Nat. Mater. 2012, 11, 422.

[44] L. Li, Y. Liu, J. Dai, A. Hong, M. Zeng, Z. Yan, J. Xu, D. Zhang, D. Shan, S. Liu, Z. Ren, J.-M. Liu, J. Mater. Chem. C 2016, 4, 5806.

[45] S. Roychowdhury, M. K. Jana, J. Pan, S. N. Guin, D. Sanyal, U. V. Waghmare, K. Biswas, Angew. Chemie Int. Ed. 2018, 57, 4043. 
[46] J. Ding, J. L. Niedziela, D. Bansal, J. Wang, X. He, A. F. May, G. Ehlers, D. L.

Abernathy, A. Said, A. Alatas, Y. Ren, G. Arya, O. Delaire, Proc. Natl. Acad. Sci. 2020, 117, 3930.

[47] Y. He, T. Day, T. Zhang, H. Liu, X. Shi, L. Chen, G. J. Snyder, Adv. Mater. 2014, 26, 3974.

[48] T. Mori, J. Martin, G. Nolas, J. Appl. Phys. 2007, 102, 073510.

[49] Z. Liu, W. Zhang, W. Gao, T. Mori, Energy Environ. Sci. 2021, Advance Article.

[50] Y. Luo, J. Wang, Y. Li, J. Wang, Sci. Rep. 2016, 6, 29801.

[51] X. Yu, H. Shao, X. Wang, Y. Zhu, D. Fang, J. Hong, 2019.

[52] T. Pandey, A. S. Nissimagoudar, A. Mishra, A. K. Singh, J. Mater. Chem. A 2020, 8, 13812.

[53] Y. Shen, F. Q. Wang, Q. Wang, Nano Energy 2020, 73, 104822.

[54] Y. Takagiwa, K. Kimura, Sci. Technol. Adv. Mater. 2014, 15, 044802.

[55] Y. Yu, M. Cagnoni, O. Cojocaru-Mirédin, M. Wuttig, Adv. Funct. Mater. 2020, 30, 1904862.

[56] X. Su, N. Zhao, S. Hao, C. C. Stoumpos, M. Liu, H. Chen, H. Xie, Q. Zhang, C. Wolverton, X. Tang, M. G. Kanatzidis, Adv. Funct. Mater. 2019, 29, 1806534.

[57] H. Kageyama, K. Hayashi, K. Maeda, J. P. Attfield, Z. Hiroi, J. M. Rondinelli, K. R. Poeppelmeier, Nat. Commun. 2018, 9, 772.

[58] Y. Pei, X. Shi, A. Lalonde, H. Wang, L. Chen, G. J. Snyder, Nature 2011, 473, 66.

[59] Y. Pei, H. Wang, G. J. Snyder, Adv. Mater. 2012, 24, 6125.

[60] W. Liu, K. C. Lukas, K. McEnaney, S. Lee, Q. Zhang, C. P. Opeil, G. Chen, Z. Ren, Energy Environ. Sci. 2013, 6, 552.

[61] L.-D. Zhao, J. He, D. Berardan, Y. Lin, J.-F. Li, C. Nan, N. Dragoe, Energy Environ. Sci. 2014, 7, 2900. 
[62] G.-K. Ren, S. Wang, Z. Zhou, X. Li, J. Yang, W. Zhang, Y.-H. Lin, J. Yang, C.-W. Nan, Nat. Commun. 2019, 10, 2814.

[63] J. Ding, B. Xu, Y. Lin, C. Nan, W. Liu, New J. Phys. 2015, 17, 083012.

[64] C. Doussier, P. Léone, Y. Moëlo, Solid State Sci. 2004, 6, 1387.

[65] C. Doussier, G. André, P. Léone, E. Janod, Y. Moëlo, J. Solid State Chem. 2006, 179, 486.

[66] C. Doussier, Y. Moelo, P. Leone, Synthesis and crystal structures of four new bromochalcogenides: $\mathrm{MnSbS2Br}, \mathrm{MnBiSe} 2 \mathrm{Br}$ and two allotropic forms of MnSbSe2Br. Crystal chemistry of the MnPnQ2XMnPnQ2X family $(P n=S b P n=S b, B i ; Q=S Q=S, S e ; X=C l X=C l$, Br, I), Vol. 8, 2006, pp. 652-659.

[67] K. Momma, F. Izumi, J. Appl. Crystallogr. 2011, 44, 1272.

[68] S. A. Sunshine, J. A. Ibers, Acta Crystallogr. Sect. C Cryst. Struct. Commun. 1987, 43, 1019.

[69] T. Tadano, S. Tsuneyuki, Phys. Rev. B 2015, 92, 054301.

[70] M. Simoncelli, N. Marzari, F. Mauri, Nat. Phys. 2019, 15, 809.

[71] W. Zhou, Y. Cheng, K. Chen, G. Xie, T. Wang, G. Zhang, Adv. Funct. Mater. 2020, 30, 1903829.

[72] B. Koley, A. Lakshan, P. R. Raghuvanshi, C. Singh, A. Bhattacharya, P. P. Jana, Angew. Chemie Int. Ed. 2021, 60, 9106.

[73] C. Yuan, J. Li, L. Lindsay, D. Cherns, J. W. Pomeroy, S. Liu, J. H. Edgar, M. Kuball, Commun. Phys. 2019, 2, 43.

[74] R. Wei, S. Song, K. Yang, Y. Cui, Y. Peng, X. Chen, X. Hu, X. Xu, J. Appl. Phys. 2013, 113, 053503.

[75] H. R. Shanks, P. D. Maycock, P. H. Sidles, G. C. Danielson, Phys. Rev. 1963, 130, 1743. 
[76] A. Jeżowski, B. A. Danilchenko, M. Boćkowski, I. Grzegory, S. Krukowski, T. Suski, T. Paszkiewicz, Solid State Commun. 2003, 128, 69.

[77] H. Y. Chen, N. Savvides, T. Dasgupta, C. Stiewe, E. Mueller, Phys. status solidi 2010, $207,2523$.

[78] D. T. Morelli, T. Caillat, J.-P. Fleurial, A. Borshchevsky, J. Vandersande, B. Chen, C. Uher, Phys. Rev. B 1995, 51, 9622.

[79] C. B. Satterthwaite, R. W. Ure, Phys. Rev. 1957, 108, 1164.

[80] Z. Tian, J. Garg, K. Esfarjani, T. Shiga, J. Shiomi, G. Chen, Phys. Rev. B 2012, 85, 184303.

[81] E. S. Toberer, A. F. May, G. J. Snyder, Chem. Mater. 2010, 22, 624.

[82] T. Caillat, J.-P. Fleurial, A. Borshchevsky, J. Phys. Chem. Solids 1997, 58, 1119.

[83] L.-D. Zhao, S.-H. Lo, Y. Zhang, H. Sun, G. Tan, C. Uher, C. Wolverton, V. P. Dravid, M. G. Kanatzidis, Nature 2014, 508, 373.

[84] K. Takahata, Y. Iguchi, D. Tanaka, T. Itoh, I. Terasaki, Phys. Rev. B 2000, 61, 12551.

[85] P. Giannozzi, S. Baroni, N. Bonini, M. Calandra, R. Car, C. Cavazzoni, D. Ceresoli, G. L. Chiarotti, M. Cococcioni, I. Dabo, A. Dal Corso, S. de Gironcoli, S. Fabris, G. Fratesi, R. Gebauer, U. Gerstmann, C. Gougoussis, A. Kokalj, M. Lazzeri, L. Martin-Samos, N. Marzari, F. Mauri, R. Mazzarello, S. Paolini, A. Pasquarello, L. Paulatto, C. Sbraccia, S. Scandolo, G. Sclauzero, A. P. Seitsonen, A. Smogunov, P. Umari, R. M. Wentzcovitch, J. Phys. Condens. Matter 2009, 21, 395502.

[86] P. Giannozzi, O. Andreussi, T. Brumme, O. Bunau, M. Buongiorno Nardelli, M. Calandra, R. Car, C. Cavazzoni, D. Ceresoli, M. Cococcioni, N. Colonna, I. Carnimeo, A. Dal Corso, S. de Gironcoli, P. Delugas, R. A. DiStasio, A. Ferretti, A. Floris, G. Fratesi, G. Fugallo, R. Gebauer, U. Gerstmann, F. Giustino, T. Gorni, J. Jia, M. Kawamura, H.-Y. Ko, A. Kokalj, E. Küçükbenli, M. Lazzeri, M. Marsili, N. Marzari, F. Mauri, N. L. Nguyen, H.-V. Nguyen, A. Otero-de-la-Roza, L. Paulatto, S. Poncé, D. Rocca, R. Sabatini, B. Santra, M. 
Schlipf, A. P. Seitsonen, A. Smogunov, I. Timrov, T. Thonhauser, P. Umari, N. Vast, X. Wu, S. Baroni, J. Phys. Condens. Matter 2017, 29, 465901.

[87] P. E. Blöchl, Phys. Rev. B 1994, 50, 17953.

[88] G. Kresse, D. Joubert, Phys. Rev. B 1999, 59, 1758.

[89] G. I. Csonka, J. P. Perdew, A. Ruzsinszky, P. H. T. Philipsen, S. Lebègue, J. Paier, O.

A. Vydrov, J. G. Ángyán, Phys. Rev. B 2009, 79, 155107.

[90] T. Tadano, Y. Gohda, S. Tsuneyuki, J. Phys. Condens. Matter 2014, 26, 225402. 
Naoki Sato,* Norihide Kuroda, Shun Nakamura, Yukari Katsura, Ikuzo Kanazawa, Kaoru Kimura, and Takao Mori

Bonding Heterogeneity in Mixed-Anion Compounds Realizes Ultralow Lattice Thermal Conductivity

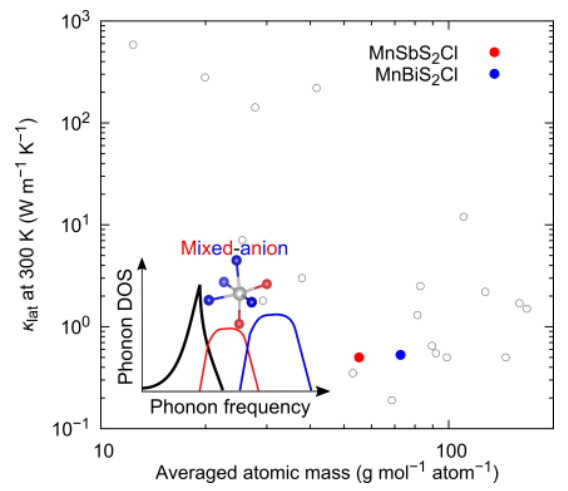

Bonding heterogeneity in chalcohalides $\mathrm{MnPnS}_{2} \mathrm{Cl}(\mathrm{Pn}=\mathrm{Sb}, \mathrm{Bi})$ derives a peak splitting of the phonon density of states, which induces a large amount of scattering phase space. Consequently, $\mathrm{MnPnS}_{2} \mathrm{Cl}$ exhibits ultralow lattice thermal conductivity $\kappa_{\text {lat }}$ despite their relatively low averaged atomic mass. Our findings will encourage the exploration of novel mixed-anion materials with lightweight and low $\kappa_{\text {lat }}$. 